\title{
BAIXA VISÃO E REABILITAÇÃO: CONHECIMENTOS DE RESIDENTES DE OFTALMOLOGIA
}

\author{
LOW VISION AND REHABILITATION: KNOWLEDGE OF OPHTHALMOLOGY RESIDENTES
}

Maria Inês Rubo de Souza Nobre ${ }^{1}$, Edméa Rita Temporini², Rita de Cássia letto Montilha', Maria Elisabete Rodrigues Freire Gasparetto ${ }^{1}$, Newton Kara-José ${ }^{2}$

\author{
'Doutora em Ciências Médicas. Oftalmologia. Faculdade de Ciências Médicas -- UNICAMP ${ }^{2}$ Docente. Faculdade de Ciências Médicas \\ - UNICAMP e Faculdade de Medicina - USP \\ CorRESPONDÊNCIA: Maria Inês Rubo de Souza Nobre \\ Rua: Moysés Lucarelli,553 Cidade Universitária. CEP: 13083500 / Campinas, SP \\ e-mail: inesnobre@fcm.unicamp.br
}

Nobre MIRS, Temporini ER, Montilha RCI, Gasparetto MERF, Kara-José N. Baixa visão e reabilitação: conhecimentos de residentes de Oftalmologia. Medicina (Ribeirão Preto) 2006; 39 (2): 253-259.

Objetivo: Verificar conhecimentos de residentes de oftalmologia sobre baixa visão e reabilitação em um hospital universitário.

Métodos: Realizou-se estudo exploratório, aplicando-se questionário sobre deficiência visual (baixa visão e cegueira), e serviços de reabilitação de deficientes visuais à amostra não probabilística formada por residentes de Oftalmologia.

Resultados: A amostra foi composta por 20 residentes que cursavam $1^{\circ}$ ano $(45,0 \%), 2^{\circ}$ ano $(30,0 \%)$ e $3^{\circ}$ ano $(25,0 \%)$; todos já haviam participado de curso teórico sobre baixa visão. A maioria revelou conhecimentos a respeito de afecções oculares que podem causar deficiência visual como: catarata, retinopatias e neuropatias $(100,0 \%)$, glaucoma, toxoplasmose e retinose pigmentar (95,0\%). Em relação à reabilitação, 100,0\% declararam ter recebido informações na residência médica. A maioria da amostra referiu conhecimento, à respeito das atividades de reabilitação sendo as mais citadas atividades de vida diária $(100,0 \%)$, locomoção $(90,0 \%)$.

Conclusão: Embora os residentes tenham declarado ter conhecimento sobre a deficiência visual e preparo teórico sobre a reabilitação, informaram deter conhecimento insuficiente em relação a algumas dessas atividades.

Descritores: Residentes. Oftalmologia. Baixa Visão. Reabilitação. Saúde Ocular.

A deficiência visual abrange cegueira e visão subnormal. Segundo a Organização Mundial de Saúde (OMS $)^{1}$ é considerado portador de cegueira o indivíduo com acuidade visual igual ou menor de $3 / 60(0,05)$, com a melhor correção óptica no olho de melhor visão, até ausência de percepção de luz, ou correspondente perda de campo visual no olho de melhor visão com melhor correção possível. A definição de baixa visão (ou visão subnormal) corresponde à acuidade visual igual ou menor do que $6 / 18(0,3)$ e igual ou maior do que 3/60 $(0,05)$ no olho de melhor visão com a melhor correção possível.

Inúmeras são as causas de perda da capacidade visual. Em geral, relacionam-se a fatores biológicos, sociais e ambientais, por vezes passíveis de serem evitados ou minimizados ${ }^{2}$.

A OMS prevê o acelerado crescimento da cegueira de um a dois milhões de casos por ano, prova- 
velmente dobrando o número total de casos ao redor do ano 2020, a não ser que sejam disponibilizados recursos suficientes para a prevenção $0^{3,4}$.

No Brasil, o número de cegos foi estimado em 0,4 a $0,5 \%$ da população, ou seja, de 4 a 5 mil pessoas por milhão de habitantes. Considerando-se a população brasileira de 160 milhões de habitantes no ano 2000 , estima-se existirem 640.000 cegos no país, tendo por critérios visão $<20 / 400(<3 / 60)^{5}$. A estimativa da prevalência de cegueira no Brasil sofre variações em termos de diferentes níveis socioeconômicos existentes em áreas mais, ou menos, desenvolvidas 5,6 . Pode-se, assim, estimar a prevalência de $0,25 \%$ em locais semelhantes a paises desenvolvidos e de $0,75 \%$ em áreas mais pobres economicamente ${ }^{5,7}$.

Necessária se faz maior interação entre médicos oftalmologistas e outros membros da equipe, a partir da qual os médicos ganhariam mais conhecimento sobre aspectos reabilitacionais da deficiência visual $^{8}$.

Reforça esse argumento o fato de que o oftalmologista acredita não ser possível, atender esses pacientes sem unir ao atendimento clínico o reabilitacional, formando-se, assim, equipe multidisciplinar ${ }^{9}$.

Nessa linha de raciocínio, a Academia Americana de Oftalmologia adotou importante medida suplementar visando à educação na reabilitação, por meio de publicações que continham informações detalhadas sobre serviços de reabilitação ${ }^{8}$.

Ademais, frequientemente os oftalmologistas têm a oportunidade de contribuir para melhorar a qualidade de vida de seus pacientes, que recebe a influência de fatores sociais. ${ }^{10,11}$. Isto ocorre com os modos de agir, em relação à prevenção e tratamento de problemas oculares ${ }^{12}$. Desconhece-se até que ponto os cursos de residência oferecem conhecimentos especializados sobre a baixa visão e reabilitação. Tendo em vista a importância do tema, os dados epidemiológicos apresentados, a magnitude do problema e a necessidade de se atuar de maneira eficaz na inclusão deste indivíduo na sociedade, este estudo teve por objetivo verificar conhecimentos em relação à baixa visão e reabilitação entre residentes de Oftalmologia.

\section{2- MATERIAL E MÉTODOS}

Foi realizado estudo exploratório aplicando-se questionário sobre deficiência visual e serviços de reabilitação a residentes de Oftalmologia atuantes no Hospital das Clínicas de Campinas e Hospital das Clínicas de São Paulo, que se dispuseram a participar da pesquisa. Formou-se amostra intencional. Foi elaborado um questionário auto-aplicável semi-estruturado e construído especificamente para a amostra do estudo (Anexos 1,2). A coleta de dados foi realizada ao final do ano de 2004, considerando o fato de que as informações sobre o tema da pesquisa deveriam ter sido incluídas no decorrer do ano. Todos os residentes receberam o questionário ao mesmo tempo, ressaltando-se a necessidade da resposta individual. Foram assegurados anonimato e sigilo dos dados. $\mathrm{O}$ instrumento foi devolvido aos pesquisadores, imediatamente após ser respondido.

\section{3- RESULTADOS}

A amostra foi composta por 20 residentes de oftalmologia que cursavam $1^{\circ}$ ano $(45,0 \%), 2^{\circ}$ ano $(30,0 \%)$ e $3^{\circ}$ ano $(25,0 \%)$; todos declararam ter estagiado no SVSN e participado de cursos sobre baixa visão.

A maioria revelou conhecimentos a respeito de afecções oculares que podem causar deficiência visual tendo apontado catarata, retinopatias e neuropatias $(100,0 \%)$, glaucoma, toxoplasmose e retinose pigmen$\operatorname{tar}(95,0 \%)$. (Tabela I).

Tabela I: Conhecimento de residentes de Oftalmologia em relação a afecções oculares que podem causar baixa visão. (Hospital Universitário, 2004)

$\mathbf{n}=\mathbf{2 0}$

\begin{tabular}{|l|r|r|}
\hline Afecções oculares * & $\boldsymbol{f}$ & $\%$ \\
\hline Catarata & 20 & 100,0 \\
\hline Retinopatias & 20 & 100,0 \\
\hline Neuropatias & 20 & 100,0 \\
\hline Glaucoma & 19 & 95,0 \\
\hline Toxoplasmose & 19 & 95,0 \\
\hline Retinose Pigmentar & 19 & 95,0 \\
\hline Miopia & 18 & 90,0 \\
\hline Estrabismo & 18 & 90,0 \\
Aniridia & 15 & 75,0 \\
\hline Oftalmia neonatal & 11 & 55,0 \\
Ceratites & 10 & 50,0 \\
\hline Olho Vermelho & 3 & 15,0 \\
Conjuntivite & 2 & 10,0 \\
Terçol & 1 & 5,0 \\
\hline * Respostas múltiplas & & \\
\hline
\end{tabular}


Em relação ao processo de reabilitação todos os respondentes declararam ter recebido informações na residência médica $(100,0 \%)$.

No que se refere às atividades desenvolvidas no processo de reabilitação, a maioria mencionou atividades apropriadas, atividades de vida diária $(100,0 \%)$, e locomoção $(90,0 \%)$. As atividades das áreas de psicologia e de fisioterapia foram menos citadas $(55,0 \%)$ (Tabela II).

Tabela II: Conhecimento de residentes de Oftalmologia em relação às atividades de reabilitação de deficientes visuais. (Hospital Universitário, 2004).

$\mathrm{n}=\mathbf{2 0}$

\begin{tabular}{|l|r|r|}
\hline Atividades * & \multicolumn{1}{|c|}{$\boldsymbol{\%}$} & \multicolumn{1}{|c|}{$\begin{array}{l}\text { Atividades específicas para } \\
\text { deficiência visual }\end{array}$} \\
\hline Orientação e Mobilidade & 20 & 100,0 \\
\hline Atividades da Vida Diária & 20 & 100,0 \\
\hline Intervenção precoce & 20 & 100,0 \\
\hline Treinamento visual & 18 & 90,0 \\
\hline Serviço social & 18 & 90,0 \\
\hline Pedagogia & 17 & 85,0 \\
\hline Terapia Ocupacional & 15 & 75,0 \\
\hline Psicologia & 11 & 55,0 \\
\hline Fisioterapia & 11 & 55,0 \\
\hline Atividades complementares & & \\
\hline Informática & 17 & 85,0 \\
\hline Educação Física & 13 & 65,0 \\
\hline Matemática & 9 & 45,0 \\
\hline Natação & 8 & 40,0 \\
\hline * Respostas múltiplas & & \\
\hline
\end{tabular}

Quanto a serviços de reabilitação, 95,0\% declararam ter tido informações no decurso da residência médica. A esse respeito, $45 \%$ dos respondentes referiram ter visitado algum serviço (Tabela III).
Tabela III: Percepções de residentes de Oftalmologia referentes a serviços de reabilitação. (Hospital Universitário, 2004).

$\mathbf{n}=\mathbf{2 0}$

\begin{tabular}{|l|r|r|}
\hline Percepções & $\boldsymbol{f}$ & $\%$ \\
\hline Refere saber da existência & 19 & 95,0 \\
\hline Refere ter visitado & 9 & 45,0 \\
\hline
\end{tabular}

\section{4- DISCUSSÃO}

Observa-se crescente número de estudos que se referem ao trabalho de oftalmologistas na área de reabilitação de deficientes visuais. Nos Estados Unidos, estudo de Waisberg ${ }^{13}$, realizado em 1984, apontou que em $50,0 \%$ das faculdades com curso de pósgraduação em Oftalmologia existia curso ou estágio regular sobre baixa visão para residentes ou pós-graduandos. Quanto à interação dos oftalmologistas com os deficientes visuais, esse estudo evidenciou que, além de haver um número reduzido de encaminhamentos de oftalmologistas para serviços de reabilitação, muitos não conheciam os serviços existentes em sua própria comunidade. No entanto, uma vez informados regularmente sobre os tipos de atendimentos fornecidos pelas instituições, ou tendo freqüentado algum curso de reabilitação para deficientes visuais, observou-se aumento do número de encaminhamentos por eles realizados ${ }^{14}$.

De forma semelhante, os sujeitos deste estudo, declararam ter recebido informações sobre deficiência visual em seu programa de residência. Provavelmente, isto também tenha colaborado para o conhecimento correto sobre as afecções oculares como causas de deficiência visual. (Tabela I).

A realização de cursos a respeito de deficiência visual junto a residentes de Oftalmologia favorece maior conhecimento das atividades de reabilitação e o conseqüente encaminhamento de casos. Esse conhecimento permite direcionar a intervenção educativa e evitar insucessos ou utilização inadequada de recursos ${ }^{15}$.

$\mathrm{O}$ conhecimento em relação às atividades de reabilitação entre oftalmologistas contribui para aumentar a efetividade desse atendimento. Assim, compete ao médico orientar o paciente para o melhor aproveitamento da visão residual. Após o atendimento e 
orientação sobre os auxílios para visão subnormal, é sua atribuição também encaminhar o paciente para os serviços de reabilitação ${ }^{13}$. Para que isto ocorra o oftalmologista necessita conhecer as especificidades e possibilidades do processo de reabilitação. A solicitação de retorno para a reavaliação e análise do seguimento das orientações inclui-se nas tarefas dos oftalmologistas.

No presente estudo verificou-se insuficiência de conhecimento dos sujeitos em relação às possibilidades oferecidas no processo de reabilitação, tais como $\mathrm{o}$ atendimento psicológico e fisioterápico (Tabela II).

São reconhecidas como atividades essenciais para a reabilitação de pessoas portadoras de deficiência visual a orientação e mobilidade, atividades da vida diária, educação básica e profissionalização ${ }^{16}$. Reforça-se a importância das atividades que promovam a eficiência visual, a utilização de recursos ópticos e não ópticos adequados às necessidades de cada indivíduo, após passar por minuciosa avaliação oftalmológica. Destaca a importância da equipe multidisciplinar no intuito de contribuir para a utilização do resíduo visual de forma contextualizada nas diferentes atividades do cotidiano $^{16}$.

No entanto, é sabido que o atendimento psicológico é fundamental para o processo de aceitação da deficiência visual, viabilizando as ações básicas da reabilitação. No que se referem à atividade fisioterápica, estudos relatam a importância dessa atividade como pré-requisito da aquisição de autonomia em orientação e mobilidade ${ }^{17}$.

É reconhecida a importância da vivência em serviços de reabilitação, para a formação dos residentes de Oftalmologia. Observa-se na Tabela III que apesar de 95,0\% declararem saber da existência de serviços, pequena parcela referiu ter visitado algum serviço.

Embora o atendimento de Visão subnormal possa estar previsto no preparo oferecido aos residentes de Oftalmologia, trata-se de matéria relativamente recente. Ademais, há que se considerar o tipo de curso oferecido, sua duração e oportunidades para a prática do conteúdo ministrado. Os resultados deste estudo indicam que ainda há muito por fazer, seja no desenvolvimento de cursos seja na realização de investigações para aprofundar o conhecimento dessa realidade.

A saúde e a qualidade de vida dependem do inter-relacionamento de múltiplos fatores psicossociais e culturais, que possibilitam ou reforçam comportamentos do indivíduo. $\mathrm{O}$ conhecimento em relação à saúde ocular constitui um desses fatores, influindo na importância que as pessoas atribuem à visão e aos cuidados para preservá-la ${ }^{2}$.

Embora os residentes tenham declarado ter conhecimento sobre a deficiência visual e preparo teórico sobre a reabilitação, informaram deter conhecimento insuficiente em relação a algumas dessas atividades.

Nobre MIRS, Temporini ER, Montilha RCI, Gasparetto MERF, Kara-José N. Low vision and rehabilitation: knowledge of Ophthalmology residents. Medicina (Ribeirão Preto) 2006; 39 (2): 253-259.

ABSTRACT: Design of the study: Observational/exploratory. Objective: To check the knowledge of ophthalmology residents on low vision and rehabilitation practices in a university hospital. Methods: An exploratory study was conducted, applying a questionnaire on visual impairment (low vision and blindness), and on rehabilitation services for visually impaired patients to a nonprobabilistic sample formed by ophthalmology residents. Results: The sample was formed by 20 residents who were studying at the $1^{\text {st }}$ year $(45,0 \%), 2^{\text {nd }}$ year $(30,0 \%)$ and $3^{\text {rd }}$ year $(25,0 \%)$, having all of them already participated in a theoretical course on low vision. A greater number of them revealed knowledge on ocular affections that can cause visual impairment such as: cataract, retinopathies and neuropathies $(100,0 \%)$, glaucoma, toxoplasmosis and pigmentary retinosis $(95,0 \%)$. As to rehabilitation practices, $100,0 \%$ declared having received information in their medical internship. Most of the sample refered, yet having knowledge about rehabilitation activities, bling daily living skills $(100,0 \%)$, locomotion $(90,0 \%)$ the most mentioned. Conclusion: Although the residents stated having knowledge of visual impairment and a theoretical preparedness, they informed they had insufficient knowledge about some of these activities.

Keywords: Residents. Ophthalmology. Low Vision. Rehabilitation. Ocular Health. 


\section{REFERÊNCIAS}

1 - World Health Organization. Global data on blindness. WHO, Geneva: WHO;1995.

2 - Armond JE, Temporini ER, Alves MR. Promoção da saúde ocular na escola: percepções de professores sobre erros de refração. Arq Bras Oftalmol 2001; 64: 395-400.

3 - Thylefors B, Négrel AD, Pararajasegaram R, Dadzie KY. Global data on blindness. Bull World Health Organ 1995;73:115-21.

4 - Resnikoff S, Pararajasegaram R. Blindness prevention programmes: past, present, and future. Bull World Health Organ 2001;79:222-6.

5 - Kara-José N, Arieta CEL. South American programme: Brazil. J Comp Eye Health 2000;13:55-6.

6 - Rodrigues MLV. Prevenção de perdas visuais. Medicina, Ribeirão Preto, 1997; 30: 84-9.

7 - Temporini ER, Kara-José N. A perda da visão - Estratégias de prevenção. Arq Bras Oftalmol 2004;67:597-601

8 - Hyvarinen L. Considerations in evaluation and treatment of the child with low vision. Am J Occup Ther 1995; 59: 891-7.

9 - Carvalho KMM. Visão subnormal: apresentação de um modelo de atendimento e caracterização das condições de diagnóstico e tratamento em um serviço universitário do Brasil. [Tese Doutorado], Campinas: Faculdade de Ciências Médicas- UNICAMP; 1993.
10 - Lee J. So you want to train in ophthalmology. Br J Hosp Med 1997; 57: 267

11 - Spivey BE. An ophthalmologist's definition of ophthalmology. Ophthalmology 1991; 98: 1877-81.

12 - Temporini ER, Kara-José N, Gondim EL, Dantas FJ. Conhecimentos sobre saúde ocular entre profissionais de um hospital universitário. Medicina, Ribeirão Preto 2002; 35: 53-61

13 - Waisberg Y. Clínica de visão subnormal: experiência inicial - particularidades do atendimento no Brasil. Rev Bras Oftalmol 1984; 42: 25-30.

14 - Greenblatt SL. Physicians and chronic impairment: a study of ophthalmologist's interactions with visually impaired and blind patients. Soc Sci Med 1988; 26: 393-9.

15 - Temporinl ER, Kara-José N. Níveis de prevenção de problemas oftalmológicos: propostas de intervenção. Arq Brás Oftalmol 1995; 58: 189-94.

16 - Organizacion Nacional de Ciegos Españoles (ONCE). El adiestramento de la vision subnormal. Madri: Grefol; 1988. 123p.

17 - Montilha RCI, Temporini ER, Kara-José N, Nobre MIRS. Deficiência visual: características e expectativas de clientela de serviço de reabilitação. Rev Ciênc Méd 2000; 9: 121-6.

Recebido em 15/08/2005

Aprovado em 13/03/2006 


\section{ANEXO I}

\section{Deficiência visual: conhecimento, opinião e conduta de residentes}

1 - Sua residência médica é em:

Oftalmologia ( )

Pediatria

Neurologia

Endocrinologia

Genética

Nefrologia

Otorrino

2 - Você está em que ano da residência:

(Ano)

3- O que você sabe atualmente sobre a deficiência visual você aprendeu de que forma: (uma ou mais respostas): Aulas/estágio em serviços de baixa visão ( )

Cursos de baixa visão em congressos

Palestras de especialistas da reabilitação

na graduação

Palestras em cursos sobre deficiência visual

Por conhecer pessoas com deficiência visual .......( )

4- Certas afecções oculares podem causar baixa visão. Assinale na lista abaixo, quais afecções você acredita serem responsáveis por baixa visão:

$\begin{array}{lrr} & \text { SIM } & \text { NÃO } \\ \text { Glaucoma } & (\text { ) } & (\text { ) } \\ \text { Terçol } & (\text { ) } & (\text { ) } \\ \text { Catarata } & (\text { ) } & (\text { ) } \\ \text { Miopia } & (\text { ) } & (\text { ) } \\ \text { Conjuntivite } & (\text { ) } & (\text { ) } \\ \text { Olho vermelho } & (\text { ) } & (\text { ) } \\ \text { Toxoplasmose } & (\text { ) } & (\text { ) } \\ \text { Oftalmia neonatal } & (\text { ) } & (\text { ) } \\ \text { Estrabismo } & (\text { ) } & (\text { ) } \\ \text { Aniridia } & (\text { ) } & (\text { ) } \\ \text { Retinose Pigmentar } & (\text { ) } & (\text { ) } \\ \text { Ceratites } & (\text { ) } & (\text { ) } \\ \text { Retinopatias } & (\text { ) } & (\text { ) } \\ \text { Neuropatias } & (\text { ) } & (\text { ( ) } \\ \text { Degeneração macular } & (\text { ) } & (\text { ) }\end{array}$

5- Na sua opinião, quais as áreas que deveriam fazer parte de um serviço de reabilitação?

Assinale as atividades específicas (AE) e as atividades complementares (AC) que deveriam estar incluídas no processo de reabilitação do deficiente visual:

Orientação e Mobilidade

AE $\quad \mathrm{AC}$

Natação

( ) ( )

Atividades de Vida Diária (AVD)

( ) ( )

( ) ( )

Pedagogia

Serviço Social

( ) ( )

( ) $($ )

Informática $\quad($ ) ( )

Psicologia $\quad($ )

Terapia Ocupacional ( ) ( )

Educação Física $\quad$ ( ) ( )

Matemática ( ) ( )

Intervenção Precoce $\quad$ ( ) ( )

Fisioterapia

( ) ( )

6- Você conhece algum serviço e/ou instituição que atende deficientes visuais?
Não
( )
Sim
( )

7- Você teve oportunidade de visitar algum serviço de reabilitação de deficientes visuais?

$\begin{array}{ll}\text { Não } & \text { ( ) } \\ \text { Sim } & \text { ( ) }\end{array}$




\title{
ANEXO 2
}

\section{Instruções para o preenchimento do questionário}

\author{
Novembro de 2004
}

Prezado Residente

Com o presente, estamos lhe entregando um questionário sobre a pesquisa "Deficiência visual: conhecimento, opinião e conduta de residentes".

Esse estudo tem por finalidade subsidiar o planejamento de um programa de ações educativas referentes às pessoas com deficiência visual.

Assim sendo, vimos solicitar sua preciosa colaboração no sentido de responder ao questionário anexo. Para tanto, solicitamos que observe as seguintes recomendações:

1. Leia com atenção, não só a pergunta, mas também todas as respostas.

2. Assinale com um $X$ a resposta que você quiser indicar.

3. Use caneta esferográfica.

4. Cada questão apresenta todas as respostas possíveis. Portanto não deixe nenhuma questão sem resposta.

Para que esta pesquisa alcance elevado grau de validade e fidedignidade, é

Necessário que ela descreva realmente o que você faz e represente sua verdadeira opinião. E, para que você possa se expressar livremente, duas providências lhe são asseguradas:

1. O questionário não é assinado.

2. Respondido, o questionário deve ser colocado em envelope.

Cordiais cumprimentos

Maria Inês Rubo de Souza Nobre e Co-autores 